\title{
Correlation of Heart Rate Variability and 24- hour Mean Heart Rate with TIMI Risk Score in Acute ST-Segment Elevation Myocardial Infarction
}

\author{
SK Chakrovortty, MR Khan, S Kundu, SK Barua, B Dutta, MN Uddin, AKMM Islam, M Ullah, AAS Majumder \\ Department of Cardiology, NICVD, Dhaka, Bangladesh.
}

Keywords:

TIMI risk score,

Heart rate

variability, STEMI.

\begin{abstract}
:
Background: Thrombolysis In Myocardial Infarction (TIMI) risk score, heart rate variability (HRV) and 24hour mean heart rate all are important predictor of prognosis after ST segment elevation myocardial infarction(STEMI). Correlation among these factors has not been studied thoroughly.

Methods: This study was conducted in NICVD (National Institute of Cardiovascular Diseases), Dhaka, from July 2008 to June 2009. Total 105 STEMI patients were included in the study. TIMI risk score were calculated and each patient under went 24hour Holter monitoring. SDNN for HRV and mean $R R$ interval for mean heart rate were recorded.

Results : Ninety one patients (mean age $53.9 \pm 10.8$ years), $86.7 \%$ were males and 14 ( mean age 59.8 \pm 8.8 years), $13.3 \%$ were female. Stratification of subjects by TIMI risk score shows that nearly $60 \%$ had risk score in the range of $3-7,17 . \%$ between $0-2$ and $24 \%$ or more than $8 . S D N N$ and $R R$ interval stratified by TIMI risk score demonstrates that both the variables decreases significantly with the increase of TIMI risk score. Among the TIMI risk groups SDNN values were 120.0 $\pm 19,871.0$ \pm 20.5 and $40.9 \pm 6.4 \mathrm{msec}$ in mild, moderate and high risk group respectively $(p=<0.001)$. Mean $R R$ were $836.8 \pm 121.0,776.7 \pm 130.3$ and $649.7 \pm 75.5 \mathrm{msec}$ in low, intermediate and high risk group respectively $(p<0.001)$. There was a significant correlation between depressed SDNN and high TIMI risk score $(r=.893, p=.001)$. High TIMI risk score also showed a negative correlation with mean $R R$ interval $(r=574, p<0.001)$. Mean $R R$ and SDNN show a perfect linear relationship $(r=0.657, p<$ $0.001)$.
\end{abstract}

Conclusion: It was observed that depressed heart rate variability and increased 24-hours mean heart rate correlates with high TIMI risk score after acute ST-elevation myocardial infarction.

(Cardiovasc. j. 2011; 4(1): 8-12)

\section{Introduction:}

Acute ST- elevated myocardial infarction (STEMI) is a major component of acute coronary syndrome and is associated with significant mortality and morbidity. ${ }^{1}$ Three small scale population based studies showed average prevalence of ischaemic heart disease 6.5 per thousand population of Bangladesh. ${ }^{2}$

Individual patient reflects a combination of clinical features that influence prognosis and these factors must be appropriately weighted to produce an accurate assessment of risk. ${ }^{3}$ Even among patients with STEMI for whom initial therapeutic options are well defined, patient risk characteristics impact short and long term medical decision making. ${ }^{4}$ The Thrombolysis In Myocardial Infarction ( TIMI) risk score for STEMI provides a convenient mechanism to identify baseline differences in risk profile and offers a framework for analysis stratified by risk group at presentation. ${ }^{5}$ The predictive value of depressed heart rate variability after myocardial infarction has been shown to be independent of other risk factors such as left ventricular ejection fraction (LVEF). ${ }^{6}$ The standard deviation of all RR intervals during 24 hours (SDNN) is by far the most common estimate of heart rate variability, and it is established as a scientifically important prognostic factor after AMI. ${ }^{7}$ Predischarge 24-

Address of Correspondence: Dr. Swadesh Kumar Chakrovortty. Department of Cardiology, NICVD, Dhaka, Bangladesh. 
hour mean heart rate is a strong predictor of mortality after myocardial infarction that can compete with LVEF and HRV. ${ }^{6}$

Thus TIMI risk score involves multiple parameters and applicable during the initial evaluation of patients. HRV study is noninvasive, safe. Analysis is computer based has good reproducibility up to several months of acute MI. ${ }^{8}$

TIMI risks core, HRV and 24-hour mean heart rate all are independent predictor of future cardiovascular events after AMI. A very few study were done to evaluate these risk factors in our country.

\section{Materials and methods:}

This cross sectional study was conducted in NICVD (National Institute of Cardiovascular Diseases), Dhaka, from July 2008 to June 2009. Informed written consent was taken from every patient or near relatives. Total 105 patients of acute STEMI were included in the study. Patients with atrial fibrillation, bundle branch block or significant noncardiac illness were excluded from the study. Out of all daily admitted patients one was taken by lottery method for final inclusion in the study.

TIMI risk score for STEMI was calculated for all patients at the time of entryin to the study considering following clinical parameters age, body weight, history of diabetes mellitus/hypertension/ angina, pulse, blood pressure, Killip class of heart failure, surface of MI, duration of symptom onset to thrombolysis. ${ }^{5}$

Subjects were divided in to three groups, 0-2 low risk, 3-7 intermediate risk and $\geq 8$ as high risk group according TIMI risk score. ${ }^{9}$

Holter monitoring for 24- hour was done for all patients in the in hospital period. Data were recorded by a recorder of dms 300-7containing compact flash card-Sandisk (64MB). The recorder was driven by single 1.5 volt AA size alkaline battery. It was attached to the seven chest lead positioned according to the mentioned colour code. All medications were continued during the recording.

Holter data were analyzed by computerized analysis method using Victor Bravo Inc.(Primium10) DSM, Nevada, USA system. Premature beats with artifacts were removed manually. Remaining data were taken as printed output. Standard deviation of the all normal to normal RR interval ie SDNN in msec. for HRV and Mean R-R interval in msec for heart rate were recorded for each patient.

SDNN $<50 \mathrm{msec}$ is considered high risk and 50$100 \mathrm{msec}$. is considered as intermediate and SDNN $>100$ considered as low risk group. ${ }^{8}$

\section{Statistical Analysis:}

All data were analyzed by using computer based SPSS (statistical programme for social science) programme. Continuous data were expressed as median or mean \pm SD. Dichotomous data was expressed as percentage. Comparison among the groups was done by unpaired t-test or ANOVA which one is applicable. The correlation test was done to find the correlation of the variables. pvalue $<0.05$ was considered as significant.

\section{Observation and Results:}

Out of 105 study subjects, 91(86.7\%) were males and the rest $14(12.3 \%)$ females giving a male to female ratio of roughly 7:1. Distribution of subjects by types of MI shows that nearly $70 \%$ had anterior MI and $24.7 \%$ had inferior MI. Very few subjects had RVI + inferior MI and other types of MI (each $2.9 \%)$ Killip class of heart failure demonstrates that $65 \%$ of the subjects was classified as Class- 1 and the rest 35\% Class-II. None of the subjects exhibited Class-III and Class-IV failure Out of 105 study subjects 103 got Streptokinase therapy. Over $70 \%$ of the patients received treatment with streptokinase after lapse 4 or more than 4 hours of time from symptom onset. The mean time lapsed between symptom onset and start of treatment with streptokinase was 5.7 hours. Risk factor distribution shows that over $50 \%$ of the subjects had hypertension, nearly $50 \%$ had smoking habit $28.6 \%$ diabetes, $12.4 \%$ family history of ischemic heart diseases, and $7.6 \%$ dyslipidaemia (Table-I)

Analysis of influence of certain biological and clinical factors on SDNN shows that diabetic MI patients had significantly depressed SDNN than that of their non-diabetic counterpart $(p=0.001)$. Older subjects (60 years or more) and patients of anterior MI had also depressed SDNN than the relatively less older subjects and patients with inferior MI, although the differences did not reach the level of significance (Table II). 
Table-I

Baseline clinical characteristics of study population $(n=105)$

\begin{tabular}{lc}
\hline Parameters & $91(87 \%)$ \\
Male & $14(14 \%)$ \\
Female & \\
Age & $53.9 \pm 10.8$ years \\
Male & $59.8 \pm 8.8$ years. \\
Female & \\
Types of MI & $70 \%$ \\
Anterior & $25 \%$ \\
Inferior & $3 \%$ \\
Inf.+RVI & $2 \%$ \\
Killip class & $68(65 \%)$ \\
I & $37(35 \%)$ \\
II & $30(29 \%)$ \\
Duration between symptom onset to thrombolysis \\
$<4$ hours & $73(71 \%)$ \\
$>4$ hours & \\
Risk factors & $52(49.5 \%)$ \\
Smoking & $53(50.6 \%)$ \\
Hypertension & $30(28.6 \%)$ \\
DM & $13(12.4 \%)$ \\
Family history of IHD & $8(7.6 \%)$ \\
Dyslipidemia &
\end{tabular}

Age $<60$ and anterior MI were found significantly prone to develop lower mean RR interval $(p=0.003$ and $p=0.009$ respectively). Diabetic MI patients exhibited a much lower mean RR interval than their non-diabetic fellows, though the difference did not turn to significant ( $p=0.100)$ (Table III).

Table-II

Influence of age, diabetes and types of MI on $\operatorname{SDNN}(n=105)$

\begin{tabular}{llc}
\hline Risk factors & \multicolumn{2}{c}{ SDNN (msec) } \\
\cline { 2 - 3 } & Mean \pm SD & P vallue \\
\hline Age & & \\
$<60$ years & $75.5 \pm 26.9$ & 0.389 \\
$>60$ years & $70.3 \pm 32.9$ & \\
Diabetes mellitus & & \\
Diabetic & $59.5 \pm 20.5$ & 0.001 \\
Non-diabetic & $77.3 \pm 32.9$ & \\
Types of MI & & 0.143 \\
Anterior & $69.3 \pm 33.1$ & \\
Inferior & $78.9 \pm 24.1$ & \\
\hline
\end{tabular}

Data were anlysed using Student's t-Test and were presented as mean $\pm \mathrm{SD}$.
Correlation between TIMI score and SDNN shows that the two variables exhibit a significantly negative correlation $(r=-0.893, p<0.001)$ (Fig. I).

Correlation between TIMI score and mean RR interval demonstrates that the two variables bear a significantly negative correlation $(\mathrm{r}=-0.574, \mathrm{p}<$ 0.001) (Fig. II). SDNN and RR interval stratified by TIMI risk score demonstrates that both the variables decreases significantly with the increase of TIMI risk score In the low risk group (TIMI 0-2) mean SDNN is $120.0 \pm 19.8$, in intermediate risk group(TIMI 3-7) it is $71.0 \pm 20.5$ and in high risk group(TIMI e"8) SDNN is $40.9 \pm 6.4 \mathrm{msec}$. Similarly mean RR interval is $\quad 836.8 \pm 121.0 \mathrm{msec}$ in low risk group, $776.7 \pm$ $130.3 \mathrm{msec}$ in intermediate risk group and $649.7 \pm$ $75.5 \mathrm{msec}$ in high risk group $(\mathrm{p}<0.001)$ (Table IV).

Correlation between mean RR and SDNN shows the two variables bear a perfect linear relationship $(r=0.657, p<0.001)$ (Fig.-3).

\section{Table-III}

Influence of age, diabetes and types of MI on mean RR interval

\begin{tabular}{lcc}
\hline Risk factors & \multicolumn{2}{c}{ Mean RR interval (m sec) } \\
& Mean \pm SD & p-value \\
\hline Age (years) & & \\
$<60$ & $728.3 \pm 116.5$ & 0.003 \\
$\geq 60$ & $807.0 \pm 147.4$ & \\
Diabetes mellitus & & \\
Diabetic & $722.9 \pm 111.2$ & 0.100 \\
Non-diabetic & $770.3 \pm 139.6$ & \\
Types of MI & & \\
Anterior & $734.6 \pm 128.4$ & 0.009 \\
Inferior & $807.4 \pm 132.5$ & \\
\hline
\end{tabular}

Data were anlysed using Student's t-Test and were presented as mean \pm SD.

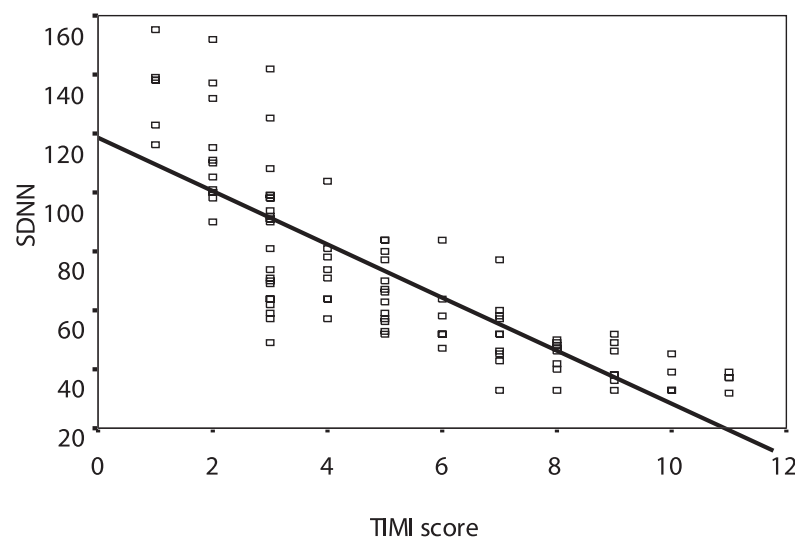

Fig.-1: Correlation between TIMI score and SDNN 
Table-IV

SDNN and RR interval stratified by TIMI risk score

\begin{tabular}{lcccc}
\hline SDNN and RR & \multicolumn{3}{c}{ TIMI score } & p-value \\
\cline { 2 - 4 } interval & $0-2$ & $3-7$ & $\geq 8$ & \\
\hline SDNN & $120.0 \pm 19.8$ & $71.0 \pm 20.5$ & $40.9 \pm 6.4$ & $<0.001$ \\
RR interval & $836.8 \pm 121.0$ & $776.7 \pm 130.3$ & $649.7 \pm 75.5$ & $<0.001$ \\
\hline
\end{tabular}

Data were analysed using ANOVA statistics and were presented as mean \pm SD.

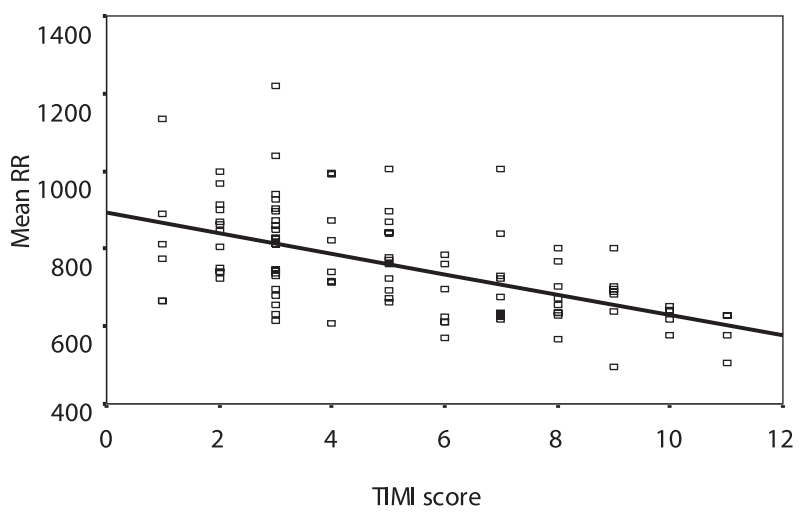

Fig.-2: Correlation between TIMI score and mean RR interval

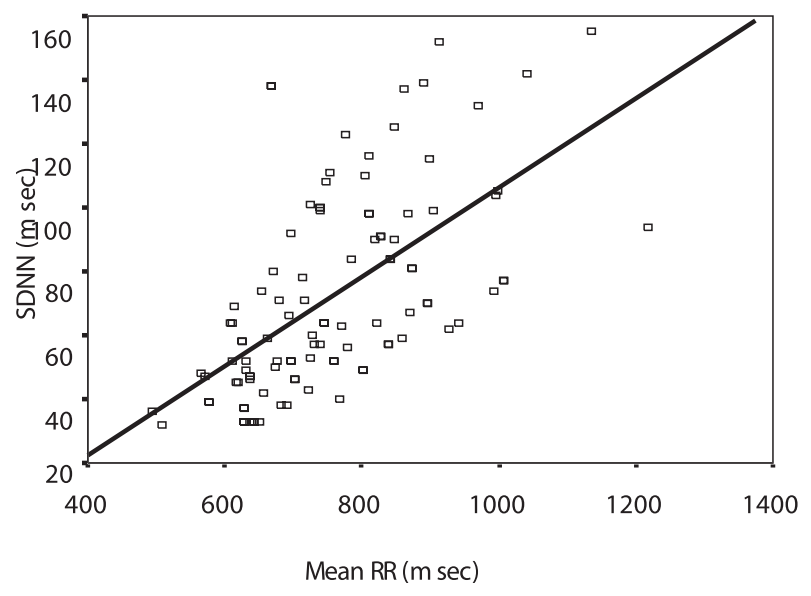

Fig.-3: Correlation between mean RR interval and $S D N N$

\section{Discussion :}

Heart rate variability (SDNN), 24 hours mean heart rate (RR interval) and TIMI risk score after acute STEMI were evaluated. Special emphasis was given to relationship of TIMI risk score with SDNN and RR interval. Factors affecting SDNN and RR interval were also evaluated.
In this study heart rate variability (SDNN) was found significantly depressed in diabetic patients than nondiabetic patients, which is consistent with the study of Ewing at al. ${ }^{10}$ as Parasympathetic damage occurs more commonly than sympathetic damage in diabetic autonomic neuropathy. This observation is not consistent with the finding of Mohosin. ${ }^{11}$

Present study showed that SDNN values are low in elderly (e"60years) age group than younger $(<60$ years) age group. Though it did not reach the level of significance this finding is consistent with the observation of Singh $\mathrm{H}$ et $\mathrm{al}^{13}$ who showed depressed HRV trend in elderly people. Mohosin ${ }^{11}$ observed similar finding from NICVD. Failure to become statistically significant may be due to small sample size and relatively few subjects over 60 years age group.

In this study HRV (SDNN) was more depressed in anterior MI group than in inferior MI group. Though the difference was not statistically significant and this is against the observation of Mohosin. ${ }^{11}$ Abildstorm et $\mathrm{al}^{7}$ proposed that relationship of HRV with site of MI is not constant. It may be changed with the duration of onset of MI to the recording of holter.

Mean heart rate (RR interval) was significantly higher in anterior MI than inferior MI in this study. This finding is corroborated by the observation of Mohosin. ${ }^{11}$ Mallani et $a{ }^{12}$ proposed that anterior ischemia may stimulate sympathetic afferent nerve and increases sympathetic tone.

Present study showed that mean heart rate was also significantly lower in older (e"6o years) age group than relatively younger $(<60$ years) age group. This finding is consistent with the data of Sing $\mathrm{H}$ et al. ${ }^{13}$ It is due to the reduced sympathetic tone associated with aging. 
In diabetic subjects mean heart rate is higher than nondiabetic subjects in this study. But the values did not reach in the level of significance $(p=0.100)$.

In present study SDNN and RR interval stratified by TIMI risk score demonstrated that both the variables decreased significantly with the increase of TIMI risk score. In the low risk group (TIMI 02) mean SDNN is $120.0 \pm 19.8$, in intermediate risk group (TIMI 3-7) it is $71.0 \pm 20.5$ and in high risk group(TIMI e"8) SDNN is $40.9 \pm 6.4 \mathrm{msec}$. So according to TIMI risk score high, intermediate and low risk group correlates with the high intermediate and low risk group according to HRV(High risk SDNN $<50$ msec, intermediate risk SDNN $<100 \mathrm{msec}$ and low risk SDNN $>100$ msec.). ${ }^{8}$

Similarly mean RR interval was $836.8 \pm 121.0$ msec in low risk group, $776.7 \pm 130.3 \mathrm{msec}$ in intermediate risk group and $649.7 \pm 75.5 \mathrm{msec}$ in high risk group $(\mathrm{p}<0.001)$ in present study. It was also consistent with the finding of Copie $\mathrm{X}^{6}$ who observed that patients with a mean RR interval $<700 \mathrm{msec}$ are at high risk of mortality after myocardial infarction.

This study showed significant negative correlation $(\mathrm{r}=-0.893, \mathrm{p}=, 0.001)$ between TIMI risk score and SDNN indicate that increase of TIMI score was associated with depressed heart rate variability.

TIMI risks core also had a negative correlation with 24 hours mean heart rate (RR interval) $(\mathrm{r}=$ $0.574, \mathrm{p}<0.001)$. That is higher TIMI risk score was associated with higher 24-hour mean heart rate in present study.

Mean RR interval has a perfect linear relationship with $\operatorname{SDNN}(\mathrm{r}=0.657, \mathrm{p}<0.001)$. This finding is consistent with the observation of Mohosin ${ }^{11}$, opie ${ }^{6}$ and Abildstrom. ${ }^{7}$

\section{Conclusion:}

It may be concluded that from the present study it was observed that depressed heart rate variability and increased 24-hour mean heart rate correlate with high TIMI risk score after acute STelevation myocardial infarction.

HRV study is noninvasive and safe. Analysis is computer based. So, HRV and 24-hour mean heart rate can be a useful tool for risk stratification of patients after acute STEMI.
Further study can be done to see whether any short term assessment of heart rate can be used for post MI risk stratification.

\section{References:}

1. Williams BA, Wright RS, Murphy JG, Brilakis ES, Reeder GS, and Jaffe AS. A new simplified immediate prognostic score for patients with acute myocardial infarction.Emergency Medicine Journal 2006 ;23:186192.

2. Islam MN, Ali MA, \& Ali M.Spectrum of cardiovascular disease: the current scenario in Bangladesh. Bangladesh Heart Journal 2004;19(1): 1-7.

3. Lee KL, Woodlief LH, Topol EJ, et al. Predictors of 30day mortality in the era of reperfusion for acute myocardial infarction: results from an international trial of 41021 patients. Circulation 1995;91:1659-68

4. Morrow D, Antman E, and Parsons L. , 'Application of the TIMI risk score for ST-elevation MI in the Nation Registry of Myocardial Infarction. JAMA 2001; 286: 1356-9.

5. Morrow D, Antman E, and Charlesworth A,TIMI risk scores for ST- elevation myocardial infarction:a convenient, bedside, clinical score for risk assessment at presentation. An intravenous nPA for treatment of infarcting myocardium early II trial sub study'. Circulation 2000; 102: 2031-7.

6. Copie X, Hnatkova K, Staunton A, Camm AJ, Marek M,Predictive Power of Increased Heart Rate Versus Depressed Left Ventricular Ejection Fraction and Heart Rate Variability for Risk Stratification After Myocardial Infarction. J Am Coll Cardiol 1996; 27: 270-6.

7. Abildstorm ZS, Jensen BT, Anger E,Pedersen CT, Nyvad O, WachtellK, Ottensen M,Kanters JK, Heart Rate Versus Heart Rate Vatiability in Risk Prediction after Myocardial Infarction. J Cardiovasc Electrophysio 2003; 14:168-173.

8. Task Force of The European Society of Cardiology and The North American Society of Pacing and Electrophysiology(1996) Heart rate variability Guidelines: Standards of measurement, physiological interpretation, and clinical use.

9. Khan SQ, Quinn P, Davies JE and Ng LL N-terminal pro-B-type natriuretic peptide is better myocardial infarction acute than TIMI risk score at predicting death after acute myocardial infarction. Heart 2008;94:40-43.

10. Ewing DJ, Neilson JM, Shapiro CM, Stewart JA, Reid W, Twenty four hour heart rate variability: effects of posture, sleep, and time of day in healthy controls and comparison with bedside tests of autonomic function in diabetic patients. Heart 1991;165:239-244

11. Mohsin MK,Heart rate variability study in patients with acute myocardial infarction. Thesis 1997 (MD, Cardiology), National Institute of Cardiovascular Diseases, Dhaka, Bangladesh.

12. Malliani A, Pagani M, Lombardi F, Sergio Cerutti S,Cardiovascular Neural Regulation Explored in the Frequency Domain. Circulation1991; 84: 482-492.

13. Singh H,Gupta G,Gupta MS, Anand JS,Aggarwal R,Verma RP, A 24 Hour Holter Study in Asymptomatic Elderly Indians.JIACM 2003; 4(4):308-14. 